LA W REN CE LIVERMORE NATIONAL LABORATORY
ZeCalc Algorithm Details

K. C. Bond, J. A. Smith, J. N. Treuer, S. G.

Azevedo, J. S. Kallman, H. E. Martz

January 3, 2013 
This document was prepared as an account of work sponsored by an agency of the United States government. Neither the United States government nor Lawrence Livermore National Security, LLC, nor any of their employees makes any warranty, expressed or implied, or assumes any legal liability or responsibility for the accuracy, completeness, or usefulness of any information, apparatus, product, or process disclosed, or represents that its use would not infringe privately owned rights. Reference herein to any specific commercial product, process, or service by trade name, trademark, manufacturer, or otherwise does not necessarily constitute or imply its endorsement, recommendation, or favoring by the United States government or Lawrence Livermore National Security, LLC. The views and opinions of authors expressed herein do not necessarily state or reflect those of the United States government or Lawrence Livermore National Security, LLC, and shall not be used for advertising or product endorsement purposes.

This work performed under the auspices of the U.S. Department of Energy by Lawrence Livermore National Laboratory under Contract DE-AC52-07NA27344. 


\title{
ZeCalc Algorithm Details
}

\author{
Karina C. Bond ${ }^{1}$, Jerel A. Smith, J. Nicolas Treuer ${ }^{2}$, Stephen Azevedo, \\ Jeffrey S. Kallman, Harry E. Martz, Jr. \\ Lawrence Livermore National Laboratory \\ Livermore, CA 94551 \\ Work performed for the \\ Science \& Technology Directorate of the \\ Department of Homeland Security \\ Statement of Work \\ IAA HSHQPM-10-X-00034
}

January 7, 2013

Version 6

(IM \# 650532)

LLNL-TR-609327
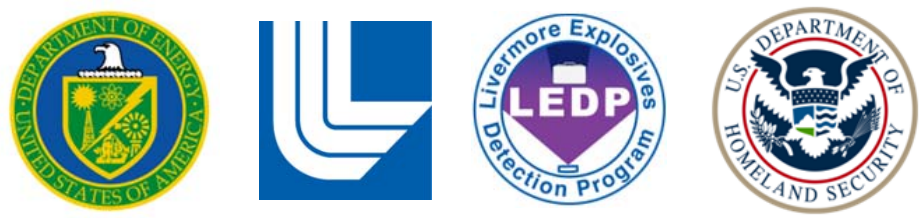

\footnotetext{
${ }^{1}$ Primary contact: Karina Carvalho Bond < bond22@llnl.gov>

${ }^{2}$ Summer LLNL intern from Wesleyan University, 45 Wyllys Avenue, Middletown, Connecticut 06459.
} 
This document was prepared as an account of work sponsored by an agency of the United States government. Neither the United States government nor Lawrence Livermore National Security, LLC, nor any of their employees makes any warranty, expressed or implied, or assumes any legal liability or responsibility for the accuracy, completeness, or usefulness of any information, apparatus, product, or process disclosed, or represents that its use would not infringe privately owned rights. Reference herein to any specific commercial product, process, or service by trade name, trademark, manufacturer, or otherwise does not necessarily constitute or imply its endorsement, recommendation, or favoring by the United States government or Lawrence Livermore National Security, LLC. The views and opinions of authors expressed herein do not necessarily state or reflect those of the United States government or Lawrence Livermore National Security, LLC, and shall not be used for advertising or product endorsement purposes.

This work performed under the auspices of the U.S. Department of Energy by Lawrence Livermore National Laboratory under Contract DE-AC52-07NA27344. 


\section{ZeCalc Algorithm Details}

Karina C. Bond, Jerel A. Smith, J. Nicolas Treuer, Stephen Azevedo, Jeffrey S. Kallman, Harry E. Martz, Jr.

Lawrence Livermore National Laboratory

Livermore, CA 94551

\section{Overview}

This report documents the implementation of the Java application ZeCalc (as released in version 3 on October 18, 2012), which calculates " $Z_{\mathrm{e}}$ ", a newly-defined "effective atomic number" for mixtures and compounds [1]. $Z_{e}$ differs from the traditional $Z_{\text {eff }}[2]$ in that it is based upon the $x$-ray cross section of the material, so it is more amenable to radiographic applications. Additionally, ZeCalc calculates $\rho_{e}$, the volumetric electron density (with units of electron- $\mathrm{mol} / \mathrm{cm}^{3}$ ), for materials with a specified composition and volumetric mass density, $\rho_{m}$ (with units of $\mathrm{g} / \mathrm{cm}^{3}$ ).

The notion of effective atomic number is especially useful in $\mathrm{x}$-ray radiography of materials. It is wellknown that $\mathrm{x}$-rays passing through an object are attenuated (scattered or absorbed) based on the $\mathrm{x}$-ray energy, the density of electrons and the x-ray cross section of the constituent atoms or molecules of the object [2]. The $x$-ray cross section (or simply cross section) of a pure element in turn depends on the $x$ ray energy and the integer atomic number, $Z$, of the element. Researchers have created large tables of cross sections for most elements over useful energy ranges [3]. Since real objects are rarely single elements-human bodies or luggage are complex mixtures of elements and molecules-and since radiography cannot resolve individual atoms anyway, we use $x$-rays to characterize macro properties of the object. The effective atomic number, then, is a kind of aggregate real number (not necessarily integer) that represents the combined Z's of all elements that the $x$-rays pass through.

The overarching concept, described in [1], is that a material with an effective atomic number, $Z_{e}$, has an (energy-dependent) $x$-ray cross section equal to a mixture of the cross sections of elements having atomic numbers immediately above and below $Z_{e}$ in the periodic chart. The two cross sections are mixed in proportion to the number of electrons contributed by each element (see Equation 1 below).

To calculate the exact cross section of a material, it would be necessary to mix the atomic cross sections of all elements present in that material in the same proportion as the numbers of atoms of each element. However, in many radiographic applications, it is necessary, efficient, or convenient to approximate the $x$-ray cross section of a mixture with a single effective atomic number. Reference [1] demonstrates that, in a broad range of situations, an excellent approximation of effective atomic number can be provided for a given radiographic application and over a limited, but specified, range of $x$-ray energies. 
This document describes the user input specifications and the underlying method ZeCalc uses to determine $Z_{e}$. Limitations on the use of $Z_{e}$ are also given here and in [1].

\section{Definitions}

The $Z_{e}$ concept provides a model to describe the $x$-ray cross sections of arbitrary materials, which could be pure elements, molecular compounds or mixtures of compounds. The $\mathrm{Z}_{\mathrm{e}}$ model is an "effective atomic number" that describes a material as a mixture of the two elements immediately above and below $Z_{e}$ in the periodic table. Therefore, $Z_{e}$ is formally defined by its electronic cross section

$$
\sigma_{Z e}\left(E_{x}\right)=(1-\text { frac }) \sigma_{e}\left(Z, E_{x}\right)+(\text { frac }) \sigma_{e}\left(Z+1, E_{x}\right)
$$

Equation 1

where "frac" is the fractional part of $Z_{\mathrm{e} .,}$ and $\sigma_{e}\left(Z, E_{x}\right)$ is the x-ray cross section for element $Z$ at energy $E_{x}$ defined below. For example, if $Z_{\mathrm{e}}=8.3, \mathrm{Z}$ is 8 and frac is 0.3 .

One can think of $\sigma_{Z e}\left(E_{x}\right)$ as a cross section model for an artificial element $\mathrm{Z}_{\mathrm{e}}$ whose exact energydependent $x$-ray cross section most closely represents the actual cross section of the material. ZeCalc is designed to find that $Z_{\mathrm{e}}$ for applications where the $\mathrm{x}$-ray signature is well-described by narrow-beam attenuation through that material; for other geometries, it is an approximation. For ZeCalc, the expression "most closely represents" means finding the best least-squares fit to the $x$-ray transmission through the material over a specified range of energies. The transmission may be evaluated with the $x-$ ray energies equally represented across that range, or may be weighted by a selection of spectra typical of the bremsstrahlung spectrum emitted by a tungsten $x$-ray source.

The ZeCalc program calculates the $Z_{\mathrm{e}}$ of a material based on the material's composition, its electron density, and the spectrum of a poly-energetic x-ray beam illuminating the material. The detector response is not considered-only the source spectrum. The method is based on the algorithm described in [1]. The program was designed to provide an effective and fast way to determine $Z_{\mathrm{e}}$ and to replace the commonly-used $Z_{\text {eff }}$ equation, originally proposed in 1937 [2], that approximates the effective atomic number as follows:

$$
Z_{\text {eff }}=\sqrt[p]{\sum_{i=1}^{N} a_{i} Z_{i}^{p}},
$$

Equation 2

where $N$ is the number of constituent elements in the material and the $a_{i}$ are the "relative electron fractions contributed by the constituent elements" [1] for each element $i$ and defined as follows:

$$
a_{i}=\frac{n_{i} Z_{i}}{\sum_{j=1}^{N} n_{j} Z_{j}}
$$

Equation 3

where $n_{i}$ is the number of atoms having $Z_{i}$, the atomic number associated with each element $i$. The exponent $p$ (set to 2.94 in Reference [2], though it varies widely), should be optimized depending on the materials and spectral region to be used [1]. The $Z_{\text {eff }}$ defined in Equation 2 does not specify the $x$-ray absorption of materials, but provides an estimate of how they should appear in $x$-ray images on a given system, relative to neighboring elements. 
In contrast, " $\mathrm{Z}_{\mathrm{e}}$ " as calculated by ZeCalc is based on the energy-dependent $\mathrm{x}$-ray cross section that best matches the transmission of a polyenergetic $x$-ray beam through a material. The program uses the chemical composition of the material, published $x$-ray cross section libraries [3] for the specified energy range, and a fitting algorithm to return the best estimate of $Z_{e}$.

Other definitions, and their units, used in this document and in ZeCalc are as follows.

- $\rho_{m}$ is the volumetric mass density of a material $\left(\mathrm{g} / \mathrm{cm}^{3}\right)$; also called "material density".

- $\rho_{e}$ is the volumetric electron density of a material (electron-mol $/ \mathrm{cm}^{3}$ ). Note that $\rho_{e}$ and $\mathrm{M}_{\mathrm{e}}$ (below) are physical properties of a specific material and are not dependent on the use of an effective-Z model.

- $M_{m}$ is the areal mass density of a material $\left(\mathrm{g} / \mathrm{cm}^{2}\right)$.

- $M_{e}$ is the areal electron density of a material (electron- $\mathrm{mol} / \mathrm{cm}^{2}$ ).

- $Z$ is the atomic number (protons/atom) for an element in the periodic table from 1 to 100.

- $A$ is the atomic weight (g/atom-mol) for an element. Values for the atomic weights were obtained from on-line NIST tabulations [4].

- $\mathrm{s}\left(E_{x}\right)$ is the x-ray incident spectrum in normalized intensity units across energy values $E_{x}$; the intensity values are normalized to 1.0 by the area under the entire spectrum from minimum to maximum energies $E_{x \min }$ and $E_{x \max }$, respectively. Detector response is not considered.

- $\sigma_{e}\left(\mathrm{Z}, E_{x}\right)$ is the total electronic x-ray cross section ( $\mathrm{cm}^{2} /$ electron-mol) of the element $Z$ for $\mathrm{x}$-ray energy $E_{x}$; these values have been tabulated from [3] and represent total electronic cross section (photoelectric, incoherent and coherent) for elements up to $Z=100$ and at each integer energy $E_{x}$ from 10 to $500 \mathrm{keV}$.

\section{ZeCalc Input Parameters}

To calculate $Z_{\mathrm{e}}$ for a particular material, the user enters or selects three pieces of information in the "ZeCalc" tab: (1) the illuminating x-ray energy spectrum $s\left(E_{x}\right),(2)$ the areal mass density $M_{m}$ that the $x-$ rays pass through, and (3) the chemical formula or elemental makeup of the material. The areal mass density, $M_{m}$, must be between 0.5 and $12.5 \mathrm{~g} / \mathrm{cm}^{2}$ inclusive. Optionally, the user can also enter (4) the material density $\rho_{m}$ from which ZeCalc can estimate the volumetric electron density, $\rho_{e}$, of the material.

\subsection{X-ray Energy Spectrum}

There are two ways to enter the x-ray energy spectrum, $s\left(E_{x}\right)$, incident to the material. One is by selecting the minimum and maximum value of the energy range (keV) of an assumed-flat $x$-ray spectrum. The minimum value of the energy range must be greater than or equal to $10 \mathrm{keV}$, the maximum value of the energy range must be less than or equal to $500 \mathrm{keV}$, and the minimum value must be less than the maximum value. The other way to enter the spectrum is to click the "Non-flat spectrum" radio button and to select from the pull-down menu one of the preprogrammed spectra typical of those used in low-energy radiography (10 to $500 \mathrm{keV}$ ). 


\subsection{Material Specification}

In the "Material Specification" section of the ZeCalc tab, the user may define the material for which Ze is calculated. Since it may consist of multiple constituent materials, there are two ways for the user to specify its composition:

1. Enter chemical formulas and weight-percentage of constituent materials, and/or

2. Enter elemental make-up and weight-percentage of constituent elements.

In the first case, to specify salt water, the user can enter a description of water and a description of salt as in Figure 1. For each material, the user must specify the weight percentage of each constituent material in the composite material. If salt accounts for $5 \%$ of the weight of a salt water mixture and water accounts for $95 \%$, then the user enters a material weight percentage of 5 for salt and 95 for water. Note that, if the percentages do not sum to 100 , the program will normalize the numbers so they do sum to 100. For example, if the numbers entered for a material are $.5, .3$ and .2 , then they will be normalized to 50,30 and 20 percent.

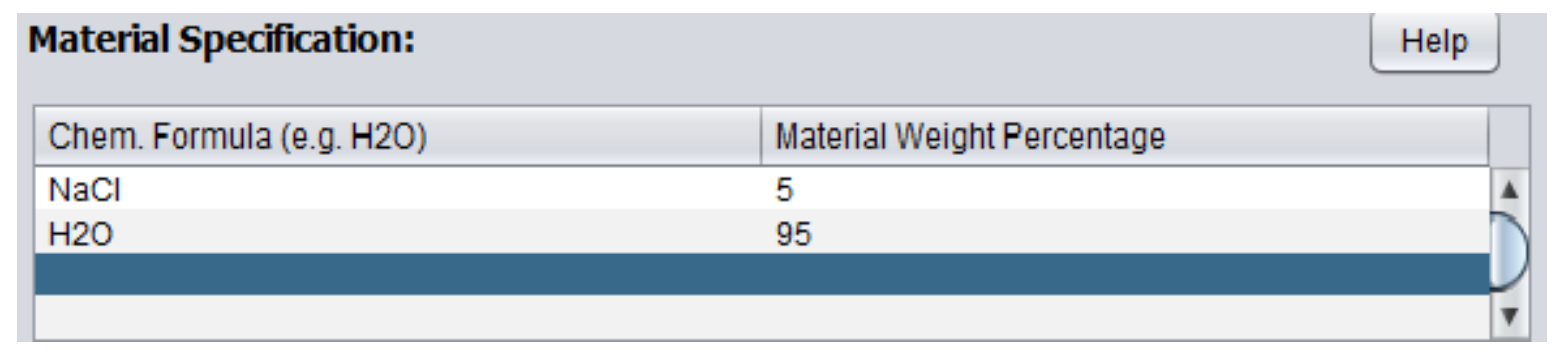

Figure 1. Material specification of salt water using chemical formulas.

The element abbreviations must appear as they do on the periodic table and are case-sensitive (Iron is $\mathrm{Fe}$, not fe or FE). Each element can have an integer multiplier within the chemical formula (e.g., H2O). Note that the user cannot enter parentheses to group elements, so each element must have its own numerical multiplier. An error message will be displayed if these constraints are not followed and the $Z_{e}$ will not be calculated.

Alternatively using case 2 , the user may choose to specify the constituent elements of the material and their elemental weight percentages as shown in Figure 2. Elements and elemental weight percentages must be entered as space-delimited sequences correspond to one another. For example, the elements of water are $\mathrm{H}$ and $\mathrm{O}$, and their weight percentages in water are 11.189 and 88.811. 


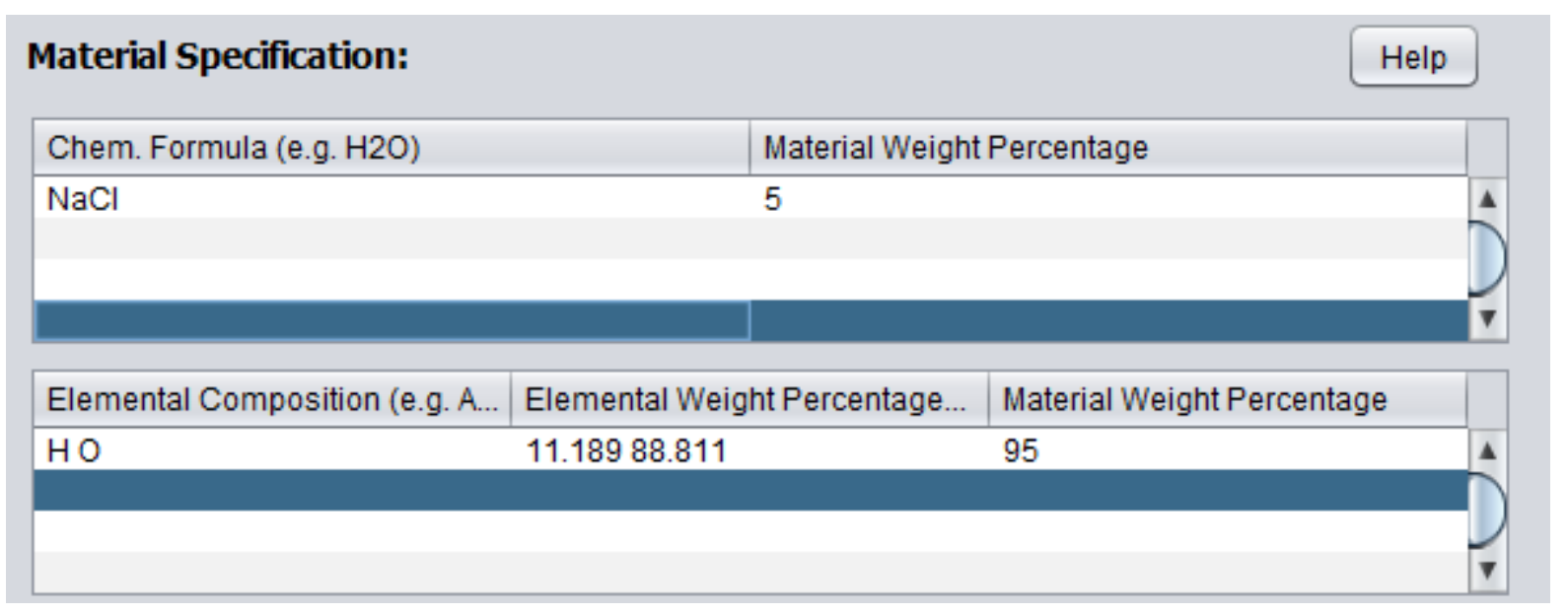

Figure 2. Material specification of salt water using chemical formula and elemental make-up.

\subsection{Conversion to Chemical Formula}

One of the first steps ZeCalc performs, after error checking, is to convert the material description into a chemical formula format that will enable the program to calculate $Z_{\mathrm{e}}$. It first converts the materials that were entered as chemical formulas into their elemental composition and their corresponding elemental weight percentages. It does this by multiplying the number after each element in the formula by the element's atomic weight, dividing it by the total weight of the material, then multiplying by 100 . Here is an example of converting $\mathrm{H}_{2} \mathrm{O}$ into its elemental composition and elemental weight percentages.

\begin{tabular}{|lll|}
\hline Element & $\begin{array}{l}\text { Atomic } \\
\text { Weight }\end{array}$ & $\mathrm{H}_{2} \mathrm{O} \longrightarrow 2 \mathrm{H}, 10$ \\
$\mathrm{H}$ & 1.00794 & Total Weight $=2(1.00794)+1(15.9994)=$ \\
$\mathrm{O}$ & 15.9994 & 18.01528 \\
& & \\
Elements: H O & Percentages: $\frac{100 * 2(1.00794)}{18.01528}, \frac{100 * 1(15.9994)}{18.01528} \longrightarrow 11.189,88.811$ \\
\hline
\end{tabular}

Second, the program multiplies each element by its "material weight percentage," which describes a particular element's weight percentage within the whole material. Third, the percent contributions of the same element, but from different materials, are summed together. Fourth, each element's percentage is divided by the element's atomic weight to calculate the ratio of the number of moles of an element compared to other elements present in the material. The ratio is normalized so that the least number of moles of an element is 1 . Basically, this gives a chemical formula for the entire material, which the main algorithm uses. Here is an example of this step: 


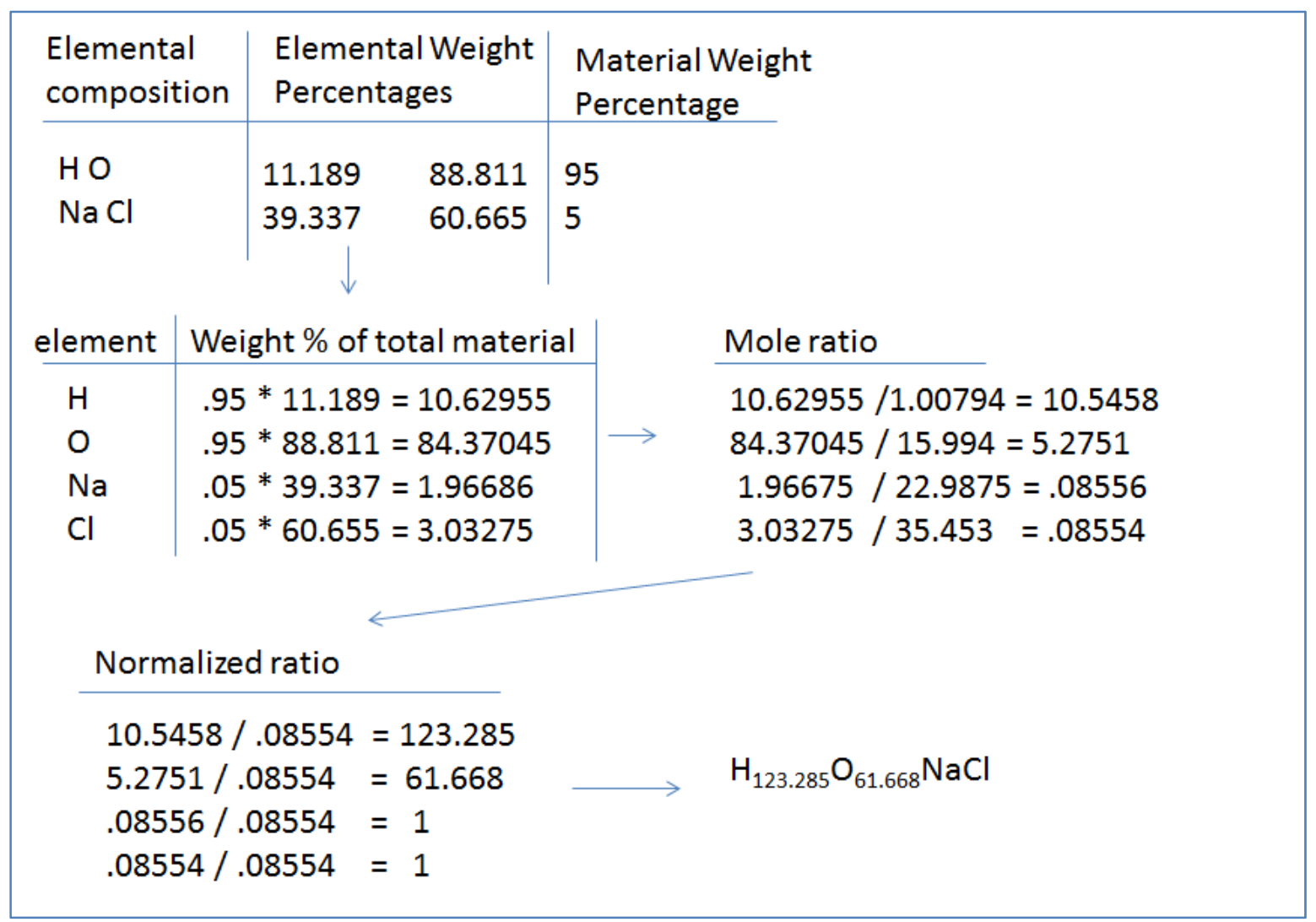

Once the normalized ratio, $r_{i}$, for each element in the material has been calculated as shown above, the weight fraction, $w_{i}$, of the $i$-th element in the material is calculated as follows

$$
w_{i}=\frac{r_{i} A_{i}}{\sum_{j=1}^{N} r_{i} A_{i}}
$$

Equation 4

where $A_{i}$ is the atomic weight for element $i$.

\section{Algorithm for Ze Calculation}

After entering the correct values into the required fields, the user presses either Enter or the "Calculate" button to initiate calculation of $Z_{\mathrm{e}}$ as shown in the screen shot of Figure 3. 


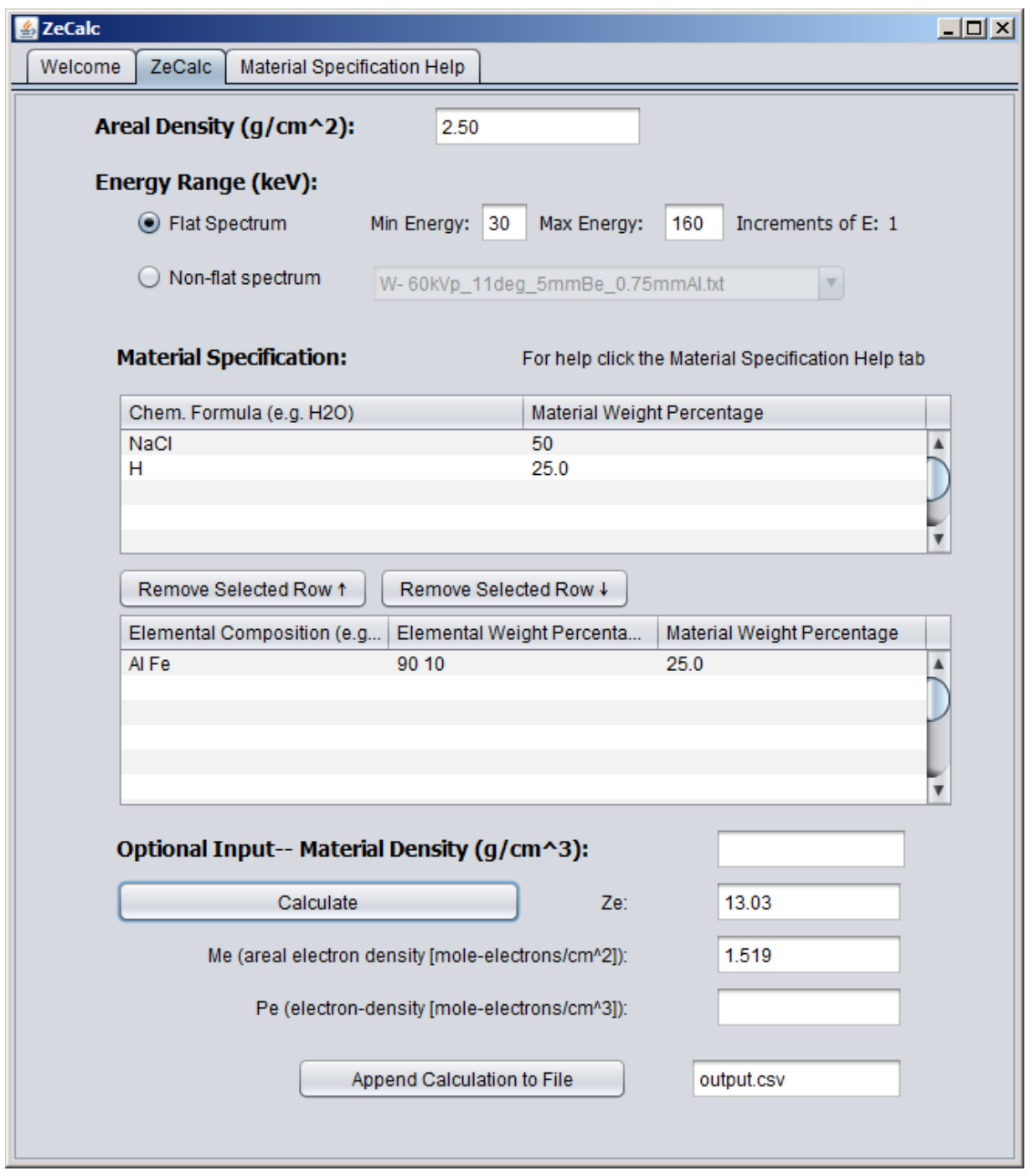

Figure 3. Screen-shot of ZeCalc. The Calculate button computes $Z_{\mathrm{e}}$.

The program checks that the inputs are within valid ranges and are in the correct formats, then computes and passes the chemical formula for the entire material to the main algorithm. This section describes the algorithmic steps ZeCalc uses to compute x-ray cross section and transmission values, a least-squares estimate of Ze, and electron densities.

\subsection{X-ray Cross section and Transmission}

ZeCalc determines the $\mathrm{x}$-ray cross section value of the material at each energy in the selected spectrum or energy-range, and defines that value to be the cross section for a fictional $Z_{\mathrm{e}}$ element. The program loads an internal cross section table containing total electronic cross section values for the first 100 elements at each integer energy from 10 to $500 \mathrm{keV}$ in units of $\mathrm{cm}^{2} /$ electron-mol. The table is extracted from the 1997 version of the Evaluated Photon Data Library [3] produced at Lawrence Livermore National Laboratory. The $Z_{\mathrm{e}}$ cross section is then defined as the sum of the fractional electronic cross section values of each element, $\sigma_{e}\left(\mathrm{Z}, E_{x}\right)$ in units of $\mathrm{cm}^{2} /$ electron-mol, as specified by the internal cross section library [3] 


$$
\sigma_{e}\left(\text { material }, E_{x}\right)=\sum_{i=1}^{N} a_{i} \sigma_{e}\left(Z_{i}, E_{x}\right)
$$

where $a_{i}$ is the electron fraction defined in Equation 3. Consider this value as the "actual" cross section of this known material, rather than the model $Z_{e}$ cross section defined in Equation 1 and estimated by ZeCalc. For example, the cross section value for water at the single energy value $E_{x}$ is determined as follows. If hydrogen and oxygen are elements 1 and 2 , the electron fractions are

$$
a_{1}=\frac{2(1)}{2(1)+1(8)}=\frac{2}{10} \text { and } \quad a_{2}=\frac{1(8)}{2(1)+1(8)}=\frac{8}{10}
$$

and the cross section for water is

$$
\sigma_{e}\left(\text { water, } E_{\chi}\right)=\frac{2}{10} \sigma_{e}\left(\mathrm{Z}_{1}, E_{\chi}\right)+\frac{8}{10} \sigma_{e}\left(\mathrm{Z}_{2}, E_{\chi}\right) \text {. }
$$

In a similar way, ZeCalc calculates the "actual" $\mathrm{x}$-ray transmission values for any specified material at each energy, following the familiar Beer-Lambert law:

$$
\frac{I\left(E_{\chi}\right)}{I_{0}\left(E_{x}\right)}=T_{Z}\left(E_{x}\right)=\exp \left(-M_{e} \quad \sum_{i=1}^{N} a_{i} \sigma_{e}\left(Z_{i} E_{\chi}\right)\right)
$$

Equation 6

where $M_{e}$ is areal electron density, also calculated by ZeCalc and defined in Section 4.3 , and $a_{i}$ is the electron fraction (unitless) for element $i$ defined as in Equation 3.

The program also calculates the x-ray cross section for any $Z_{\mathrm{e}}$ model, $\sigma_{z_{e}}\left(E_{x}\right)$, described in Equation 1. Then the transmission for $Z_{e}$ at each energy can be calculated as follows:

$$
\frac{I\left(E_{\chi}\right)}{I_{0}\left(E_{x}\right)}=T_{Z e}\left(E_{x}\right)=\exp \left(-M_{e} \sigma_{z_{e}}\left(E_{x}\right)\right)
$$

Equation 7

The technique for determining $Z_{\mathrm{e}}$ for an arbitrary material is based on a least-squares-fit criterion described in the next section.

\subsection{Calculating $\mathrm{Z}_{\mathrm{e}}$ by Least Squares}

The iterative least squares method is used to solve for $Z_{e}$ by minimizing the error between the actual transmission of the material $T_{Z}$ and the $Z_{\mathrm{e}}$ model transmission $T_{Z e}$. The precise definition of the error is given below.

As an initial approximation for $Z_{\mathrm{e}}$, the algorithm uses $Z e_{0}=Z_{\text {eff }}$ from Equation 2. The algorithm iterates on the following four steps, incrementing $i$ until meeting the stopping criteria in Step 4.

Step 1: Calculate the transmission $T_{Z e 1}, T_{Z e_{2}}, T_{Z e 3}$ using Equation 7 for $Z e_{1}, Z e_{2}, Z e_{3}$ where

$$
\begin{aligned}
& Z e_{1}=Z e_{(i-1)}-\Delta Z e \\
& Z e_{2}=Z e_{(i-1)} \\
& Z e_{3}=Z e_{(i-1)}+\Delta Z e
\end{aligned}
$$


and where $\Delta Z e=0.01$.

Step 2: Calculate the error $\epsilon_{j}$ for the three values of $T_{Z e}$ in Step 1 from the actual transmission, $T_{Z}\left(E_{x}\right)$, in Equation 6. The error in the transmission for each trial $Z_{\mathrm{e}}$ is the sum of the squared difference between the $\mathrm{Z}_{\mathrm{e}}$-model and actual transmission values at each energy across the energy range:

$$
\epsilon_{j}=\sum_{E x=E x \min }^{E x \max } s\left(E_{x}\right)\left[T_{Z e j}\left(E_{x}\right)-T_{Z}\left(E_{x}\right)\right]^{2}, \quad 1 \leq j \leq 3,
$$

Equation 8

where $s\left(E_{x}\right)$ is the normalized intensity of the energy spectrum at energy $E_{x}$, and where $E_{x m i n}$ and $E_{x \max }$ are the minimum and maximum energies in the spectrum.

From this error, the program has three two-dimensional points of the form $\left(Z e_{j}, \epsilon_{j}\right)$. With these three points, the program solves the following system of equations for $a, b$ and $c$ :

$$
\begin{aligned}
& \epsilon_{1}=a\left(Z e_{1}\right)^{2}+b\left(Z e_{1}\right)+c \\
& \epsilon_{2}=a\left(Z e_{2}\right)^{2}+b\left(Z e_{2}\right)+c \\
& \epsilon_{3}=a\left(Z e_{3}\right)^{2}+b\left(Z e_{3}\right)+c .
\end{aligned}
$$

Equation $9 a$

Equation $9 b$

Equation 9c

Step 3: Calculate the $Z_{\mathrm{e}}$ at the minimum error assuming the error is parabolic in $Z_{\mathrm{e}}$. ZeCalc solves the above three equations with a quadratic error function dependent on $Z e_{j}$. By setting the derivative to zero, we see that the $Z_{\mathrm{e}}$-value at the relative minimum of the function - the $Z_{\mathrm{e}}{ }^{-}$ value with the least error-is

$$
Z e_{\min }=-\frac{b}{2 a}
$$

Equation 9d

$Z e_{\min }$ in Equation $9 \mathrm{~d}$ is the initial approximation for the next iteration in the least squares algorithm

$$
Z e_{(i)}=Z e_{\min }
$$

Step 4: Evaluate the termination criteria. The least squares algorithm terminates when the difference between the $Z e_{\text {min }}$ of the current iteration and the previous iteration is less than .001 , or when the number of iterations reaches 25; i.e., when $\left|Z e_{(i-1)}-Z e_{(i)}\right|<0.001$ or when $i \geq 25$. The current $Z e_{\min }$ at termination is output as the $\mathrm{Z}_{\mathrm{e}}$.

\subsection{Calculating Areal and Volumetric Electron Densities}

In addition to $Z_{e}$, the $Z$ eCalc program also computes the areal electron density, $M_{e}$, from the areal material density entered by the user, using the formula 


$$
M_{e}=M_{m} \sum_{i} w_{i} Z_{i} / A_{i}
$$

If the user has entered the volumetric material density, $\rho_{m}$, then the electron density, $\rho_{e}$, will be calculated using

$$
\rho_{e}=\rho_{m} \sum_{i} w_{i} Z_{i} / A_{i},
$$

Equation 11

where the weight fractions, $w_{i}$, are defined in Equation 4.

The $M_{e}$ and (and if applicable $\rho_{e}$ ) values are displayed along with the $Z_{e}$ in the main ZeCalc display.

\section{Other Features and Limitations}

With the current version of ZeCalc, when the user presses Enter or the Calculate button, three plots are generated and displayed (Figure 4$)$. The first is the normalized $x$-ray spectrum used to calculate $Z_{\mathrm{e}}$. The next plot displays $x$-ray transmission versus energy values for the actual material $T_{Z}\left(E_{x}\right)$, and for the calculated $\mathrm{Z}_{\mathrm{e}}$ model of the material $T_{Z e}\left(E_{x}\right)$. These are graphical representations of the transmission of the $x$-rays through the material at each energy as calculated in Equations 6 and 7, respectively. These two curves are often so similar that it is difficult to distinguish them, so the final plot displays the difference values versus energy.

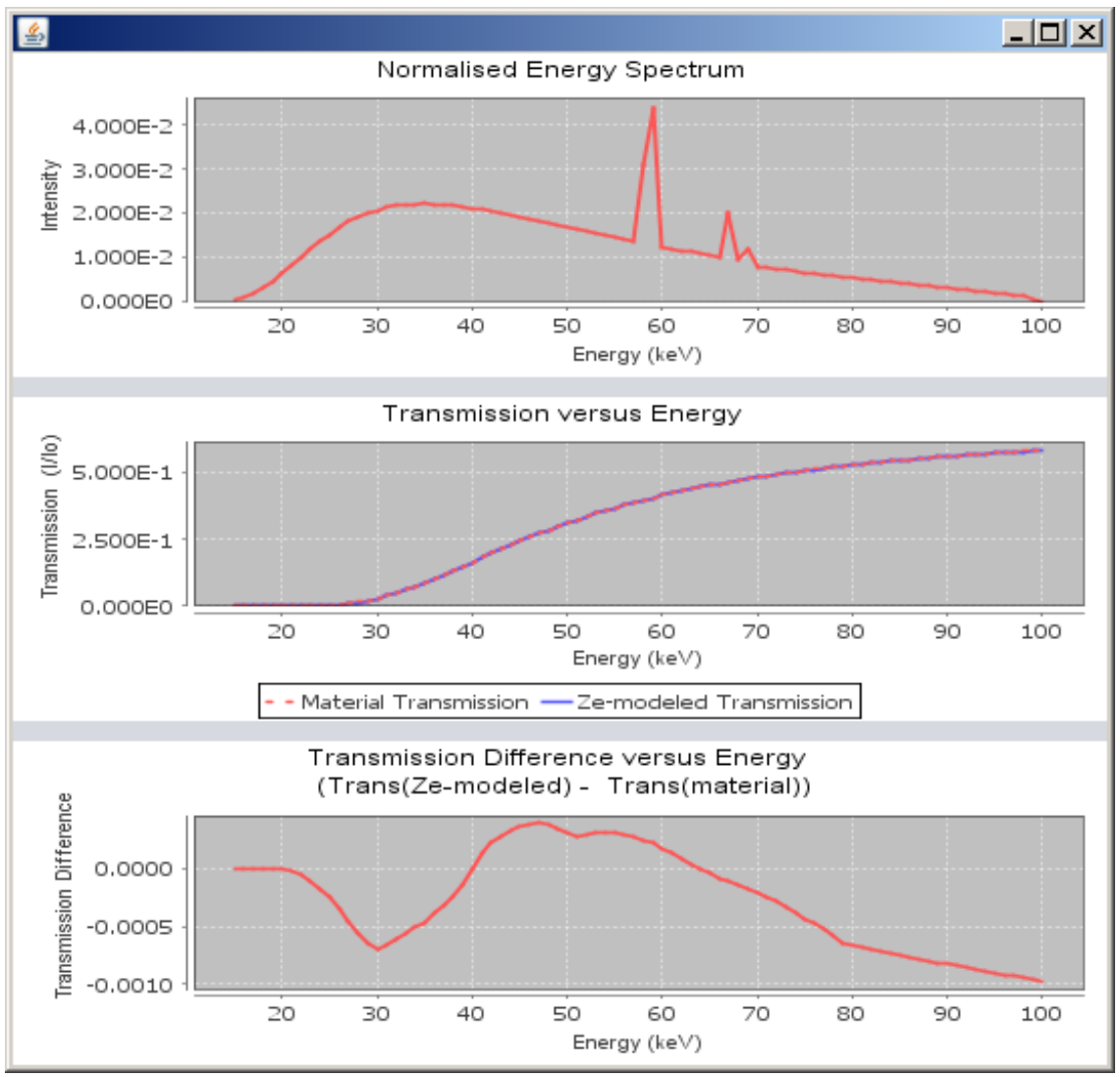

Figure 4. Plots generated by ZeCalc of the $x$-ray spectrum and the transmission fit of $Z_{e}$. 
If the two transmission curves are very different (difference values are large), we do not recommended using the calculated $Z_{\mathrm{e}}$ for that material/spectrum combination. It is important to note that, for materials with prominent absorption-edges present in the $x$-ray cross section for the energy range of interest (typically high-Z materials with $Z>50$ ), any "effective- $Z$ " model-whether $Z_{e}$ or $Z_{\text {eff }}$ or any othercannot accurately describe the $x$-ray transmission properties, and should therefore not be used. If there are prominent absorption edges (discontinuous jumps) in the red-dotted curve of the x-ray transmission spectrum, then an effective- $Z$ model is not appropriate.

Also, ZeCalc records the session history. The user can press the "Append Calculation to File" button and the program will append the inputs and outputs for the last calculation to a ".csv" file so that the data can be read into a spreadsheet program. ZeCalc will not erase previously appended calculations. The name of the file can be defined by the user.

\section{References}

[1] Smith J A, Martz H E and Kallman J S, "Case for an Improved Effective-Atomic-Number for the Electronic Baggage Scanning Program," Lawrence Livermore National Laboratory Technical Report, LLNLTR-520312, Dec 2011.

[2] M. V. Mayneord, "The Significance of the Roentgen," Acta of the International Union Against Cancer, V ii, p. 271, 1937.

[3] D.E. Cullen, J.H. Hubbell, and L. Kissel, “EPDL97: The Evaluated Photon Data Library version '97," Lawrence Livermore National Laboratory Technical Report, UCRL-50400-VOL-6-REV-5, Sept 1997.

[4] Atomic Weights were taken from the NIST site at: http://www.nist.gov/pml/data/comp.cfm 